\title{
Management of Lower Limb Soft Tissue Sarcomas with Major Neurovascular Involvement: Current and Future Perspectives
}

\author{
Authors: \\ Noor Tamimi, ${ }^{*}{ }^{*}$ Hussein Sweiti² \\ 1. Rothman Orthopedic Institute at Thomas Jefferson University, Philadelphia, \\ Pennsylvania, USA \\ 2. Janssen Research \& Development, Spring House, Pennsylvania, USA \\ *Correspondence to hsweiti@gmail.com
}

Disclosure: $\quad$ Tamimi has declared no conflicts of interest. Sweiti is currently an employee of Janssen, a pharmaceutical company of Johnson \& Johnson; the creation of this article is not related to or supported by the author's employer.

Acknowledgements: Tamimi searched and reviewed the literature, significantly contributed to writing and correction of the review. Sweiti developed the concept for the article, reviewed the literature, and contributed to writing and correcting the review.

Received: $\quad 05.04 .20$

Accepted: $\quad 25.08 .21$

Keywords: $\quad$ Lower limb, multidisciplinary management, neurovascular involvement, sciatic nerve, soft tissue sarcoma (STS).

Citation:

EMJ Oncol. 2021;9[1]:91-99.

\begin{abstract}
Lower limb soft tissue sarcomas are a group of rare mesenchymal tumours that may grow in close anatomical proximity to major neurovascular structures, leading to significant oncological and surgical challenges for treating physicians. This article reviews the current literature on the multidisciplinary approach of treating lower limb soft tissue sarcomas with neurovascular involvement and describes the increasing shift towards limb-sparing surgeries, with an emphasis on improved functional outcomes based on a multimodal treatment approach. In addition to identifying the histological subtype of the tumour, classifying the neurovascular involvement precisely is key in planning the appropriate treatment. Existing classification systems for both vascular and neural involvement are discussed, and a combined neurovascular classification is proposed together with a general treatment algorithm.
\end{abstract}

\section{INTRODUCTION}

Soft tissue sarcomas (STS) are a group of rare mesenchymal tumours that can arise in patients of any age and in a variety of anatomic sites. ${ }^{1}$ It is estimated that STS make up $1 \%$ of all adult malignancies and that their incidence is on the rise. ${ }^{2,3}$ The lower limb is the most commonly affected site, with approximately $28 \%$ of STS arising there. ${ }^{4}$ With at least 50 different histologic subtypes, STS are considered highly heterogenous in terms of their histopathology and their tendency to metastasise. ${ }^{5}$ Undifferentiated pleomorphic sarcomas and liposarcomas are the most common histopathologic subtypes in adult patients. ${ }^{5}$

STS may arise from or grow towards neighbouring vascular and neural structures, leading to significant oncological and surgical challenges. In these cases, the responsible surgeon must 
find a balance between the need for a complete tumour resection, with microscopically negative margins, and the desire to limit the invasiveness of the operation and minimise long-term disabilities. In the past, the involvement of major neurovascular structures of the lower extremity was often associated with limb amputations and debilitating surgeries. ${ }^{6}$ However, current multimodal treatment strategies pursue the goal of limb preservation, whilst minimising the risk of local or systemic disease recurrence.

There are limited data on the frequency and typical localisation of neurovascular involvement in STS. It appears that the inguinal region, the medial thigh compartment, and the popliteal fossa are common sites of lower limb STS with vascular involvement. ${ }^{7}$ The reported frequency of major vascular involvement varies between 5\% and $10 \%$ of all adult patients with STS of the lower extremity, with femoral vessels being reported as the most commonly involved vascular structures followed by the inguinal and popliteal vessels. ${ }^{8-10}$ En bloc tumour resection, with resection of the great vessels, has been reported in up to $5.0 \%$ of all patients with lower extremity sarcomas. ${ }^{711-13}$ In contrast, nerve resection was carried out in only $1.2 \%$ of cases of lower limb STS with neural involvement, according to a study by Brooks et $a{ }^{14}$ In a recent study, however, sciatic nerve involvement was reported at $15.0 \%$ of all lower limb STS, with $4.5 \%$ of all cases of lower extremity STS requiring complete nerve resection. ${ }^{15}$

In this article, the authors aim to review the current literature regarding the multidisciplinary management of lower limb STS, with a focus on diagnostic and therapeutic management strategies of major neurovascular involvement. They also aim to suggest aspects for future research to further assess the role of a limbpreserving multimodal therapeutic approach.

\section{DIAGNOSTIC STRATEGIES}

\section{Selection of Imaging Studies}

Following the initial clinical examination of a suspicious lesion, further imaging is often necessary in establishing the diagnosis. ${ }^{16}$ An initial ultrasound examination of the lesion may be helpful in determining its size and relationship to the fascia. ${ }^{17} \mathrm{MRI}$ remains the imaging modality of choice for diagnosing soft tissue lesions, providing useful anatomical details necessary in planning the surgical tumour resection. ${ }^{17}$ Gadolinium enhancement of the MRI examination is often necessary in determining the vascularity of the tumour and its anatomical relation to blood vessels and nerves. ${ }^{16} \mathrm{CT}$ imaging can alternatively be used when an MRI examination is contraindicated. The use of conventional angiography or duplex sonography in addition to magnetic resonance angiography may also be necessary in STS with vascular involvement. ${ }^{8}$ Tumour-induced anatomical changes in surrounding vessels can be shown using digital subtraction angiography. ${ }^{7}$ PET scanning is being increasingly employed as an imaging modality in the investigation of STS. In addition to screening for metastatic disease, particularly lymph node involvement, fluorodeoxyglucose PET scans may also be used as a prognostic tool in patients with STS due to the demonstrated correlation between tumour grade and fluorodeoxyglucose uptake. 18,19

The pre-operative radiological imaging and intra-operative findings ultimately determine whether and to what extent a nerve or vessel resection is necessary. ${ }^{8}$ Pre-operative staging of the tumour is essential when planning the surgical tumour resection and the surgical margins. ${ }^{20}$ The gold standard for local staging of the tumour is MRI examination. ${ }^{17}$ When screening for metastatic disease, radiographic or CT imaging of the lungs is essential as STS primarily metastasise to the lungs. ${ }^{17}$

\section{Tumour Biopsy and Histological Confirmation}

When planning the appropriate course of treatment, and particularly the choice of neoadjuvant or adjuvant radiation or chemotherapy, the initial accurate tumour diagnosis and identification of the correct histopathologic subtype is paramount. ${ }^{20}$ Due to the high rate of misdiagnoses, with reported rates of up to $30 \%$, a reference pathological examination to confirm the tumour histopathology, particularly in community pathology, is highly recommended. ${ }^{21}$

A biopsy of the tumour is usually the first step in accurately identifying its histopathological subtype. The biopsy is usually obtained via an open biopsy as the diagnostic accuracy of the histologic cell type and grade of an open biopsy 
is superior to that of a needle biopsy, despite the lower rate of complications associated with needle biopsies. ${ }^{22}$ An MRI of the tumour should be performed prior to the open biopsy to avoid traumatic injury of the surrounding tissues and for a qualitatively superior interpretation of the MRI images. ${ }^{17}$ When performing an open biopsy of the tumour, the incision should be longitudinal and be in line with future surgical incisions. ${ }^{17}$ A transverse incision in the extremities and the exposure of neurovascular structures should always be avoided.23,24 It is also recommended that the surgeon who is planning to perform the definitive tumour resection also carries out the biopsy of the tumour to ensure the correct placement of the incision. ${ }^{20}$

\section{Classification of Vascular Involvement}

Schwarzbach et al. ${ }^{8}$ proposed a four-stage classification system and treatment algorithm for extremity STS with vascular involvement. Arterial and venous tumour invasion of the great vessels is classified as Type I vascular involvement. These tumours are treated with an en bloc tumour resection, along with resection of the involved vessels followed by an arterial reconstruction. A venous reconstruction is not necessary when the greater saphenous vein is patent, enabling a collateral venous drainage. Type II vascular involvement refers to tumours with arterial encasement, attachment, or infiltration, which are treated with arterial and tumour resection followed by arterial reconstruction. Tumours that only involve the great veins are classified as Type III and are treated with a resection of the tumour and the involved veins. As with Type I tumours, a venous reconstruction is only required if the collateral venous drainage is impaired. STS of the extremities without vascular involvement are classified as Type IV and are treated with marginal resection of the tumour without vascular resection. This classification system and treatment algorithm has been summarised in Figure $1 .^{8}$ The histopathological proof of tumour vessel infiltration, in addition to tumour grade and margin of resection, has been demonstrated as a negative predictor of survival. ${ }^{8}$ Tumour grade and resection margins were also found to be important prognostic factors for survival and development of metastases in patients with lower limb STS with sciatic nerve involvement. ${ }^{15}$

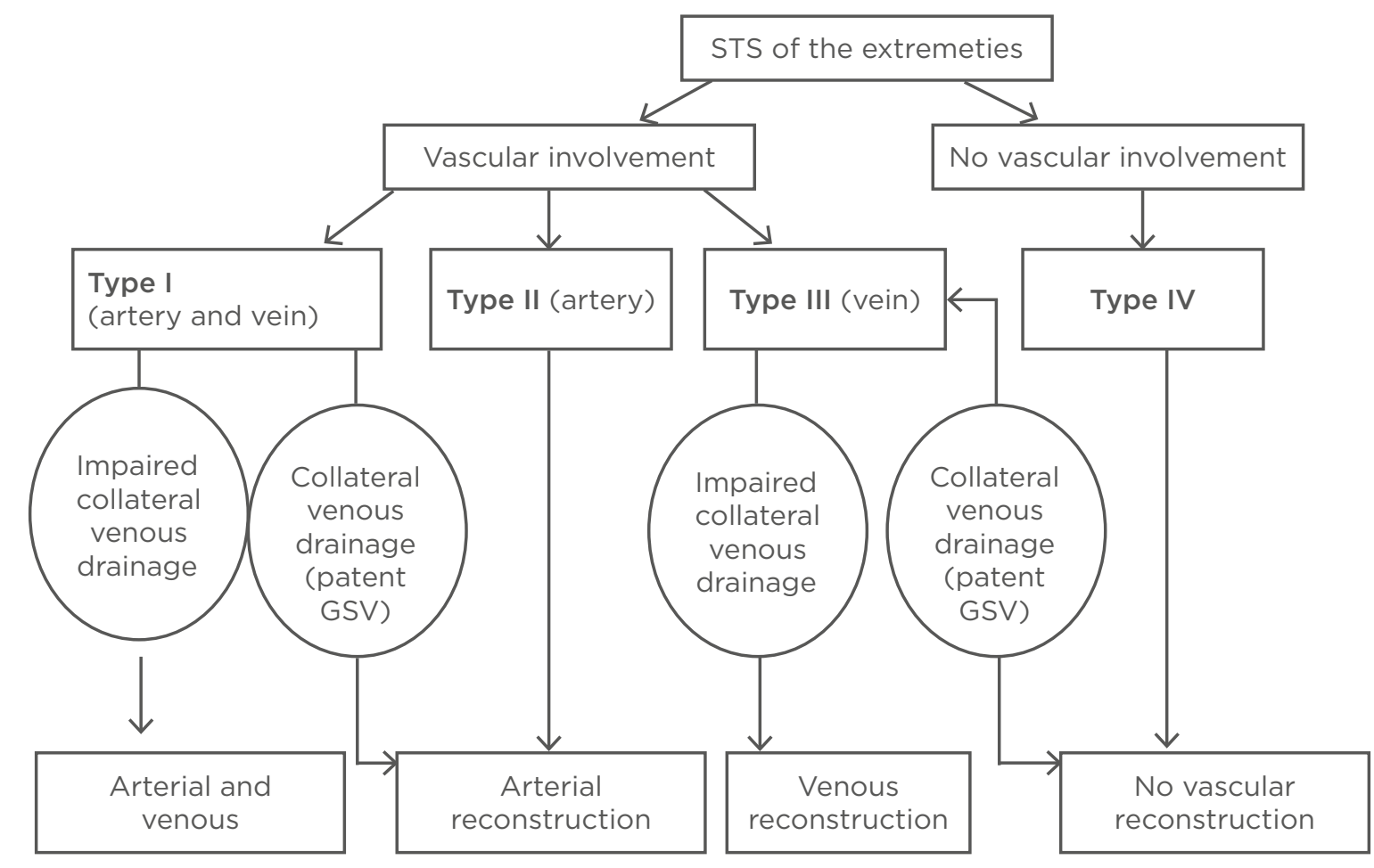

Figure 1: Classification of vascular involvement and treatment algorithm for patients with soft tissue sarcomas of the extremities. ${ }^{8}$

GSV: great saphenous vein; STS: soft tissue sarcomas. 


\section{Classification of Neural Involvement}

A recent study by Sweiti et al..$^{15}$ classified lower limb STS with sciatic nerve involvement into three main categories. Type A tumours were referred to as $\geq 180^{\circ}$ tumour encasement of the nerve based on MRI or CT imaging. These tumours were reassessed intra-operatively by either visually analysing and palpating the relationship of the nerve to the tumour where possible or visualising the extent of nerve contact with ultrasound guidance. If a Type A tumour was intra-operatively confirmed, patients underwent en bloc compartmental resections together with the nerve. STS with direct nerve contact $\left(<180^{\circ}\right)$ were classified as Type B and underwent compartmental resections of the tumour with an epineural nerve dissection. STS without nerve involvement were classified as Type $C$ and were treated with a tumour resection without nerve dissection or resection. This classification of lower limb STS with neural involvement and the suggested treatment approach are summarised in Figure $2 .^{15}$

\section{MULTIMODAL TREATMENT STRATEGIES}

The treatment of lower limb STS with neurovascular involvement presents a unique challenge, which requires multidisciplinary management and close co-ordination between surgical, medical, and radiation oncologists. The ultimate goal for patients with non-metastatic STS should be to maintain long-term, diseasefree survival, while keeping limitations of limb function to a minimum. Due to the inferior outcomes demonstrated in patients receiving surgical interventions, including biopsies, prior to referral to a multidisciplinary centre, early referral to a specialist centre is strongly recommended to ensure an optimal holistic treatment strategy, which has been associated with improved patient outcomes. ${ }^{23-27}$

\section{Limb-Salvage Surgery}

There has been a continuous shift towards multimodal treatment and preservation of limb function following the results of the National Cancer Institute's $(\mathrm{NCl}$ ) randomised prospective study in 1982, which found no significant difference in the survival rates of patients with STS of the extremities when comparing limb amputation with limb-sparing surgery combined with radiation therapy. ${ }^{28,29}$ Limb-sparing surgery with resection of the sciatic nerve in STS of the lower extremity was first reported in 1984, with the hypothesis that the use of ankle-foot orthoses leads to superior functional outcomes when compared to hip disarticulation. ${ }^{30}$

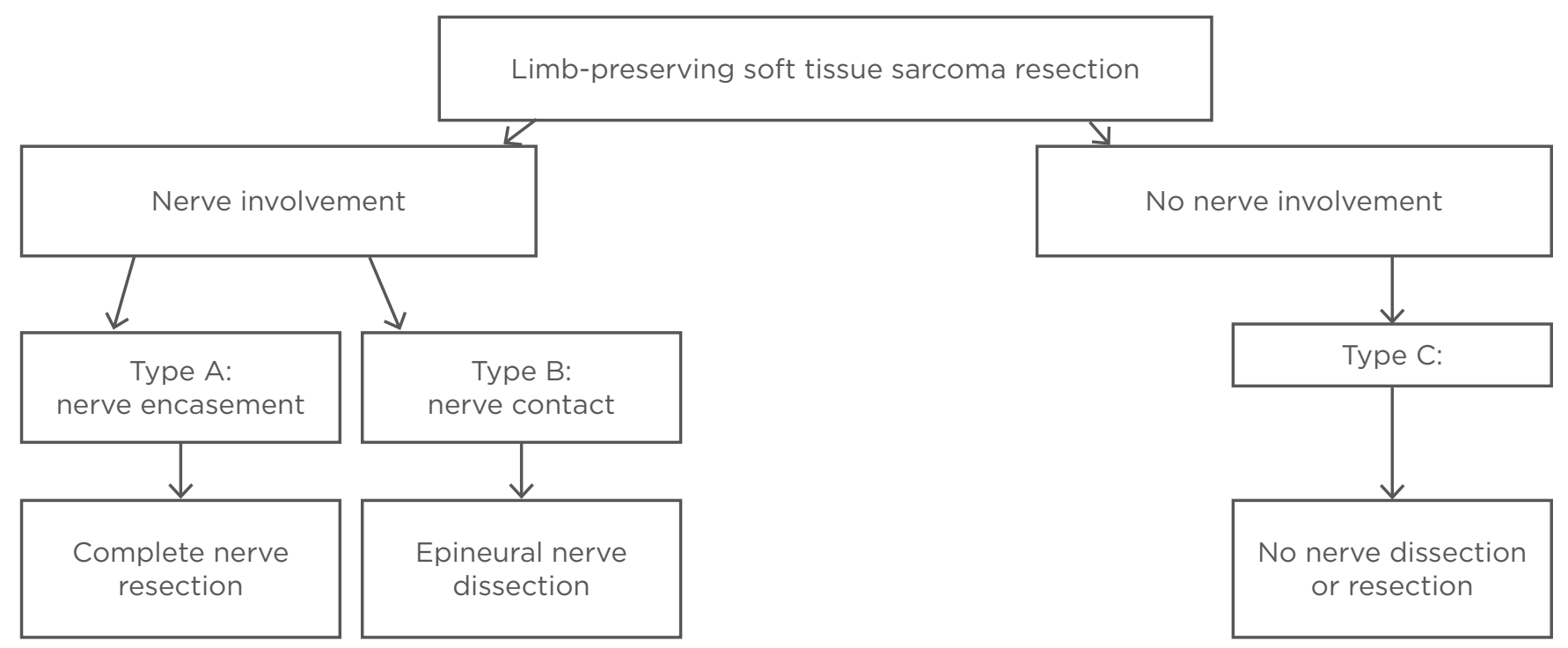

Figure 2: Classification of sciatic nerve involvement and surgical treatment algorithm for lower limb STS. ${ }^{15}$ 
Tumour involvement of the great vessels or nerves in the lower extremity was previously an indication for limb amputation, ${ }^{6}$ but more recent studies have shown comparable oncological outcomes and superior functional outcomes in limb-sparing surgery with vessel reconstruction ${ }^{8,9,31-34}$ and partial or complete sciatic nerve resection. 14,15,35-37 Limb-sparing surgery is, therefore, considered the current standard of surgical treatment for STS of the lower extremity. ${ }^{8}$ Currently, acknowledged contraindications for limb-sparing surgery include an expected patient survival of $<3$ months, the presence of a pathological fracture, local or systemic sepsis, as well as a significant contamination of adjacent tissues due to poorly performed biopsies or excisions. ${ }^{20}$

Local disease control is essential for diseasefree survival, with surgical resection being the only treatment modality capable of achieving a local disease-free state. ${ }^{38}$ The risk of a local recurrence is significantly reduced in tumour resections with wide margins. ${ }^{39}$ Obtaining wide, microscopically negative margins is, however, particularly challenging if the tumour is in close contact with major neurovascular structures..$^{15} \mathrm{~A}$ marginal margin of 1-2 $\mathrm{mm}$ is, therefore, generally accepted when trying to preserve functional tissue, such as when dissecting a major nerve. ${ }^{38}$

Due to the growth pattern of STS, it is more important to achieve wider margins when excising the tumour longitudinally when compared to the transverse excision during surgical tumour resection. ${ }^{38}$ Drains are usually placed in line with the skin incision and exit distally, which is important for any necessary future surgeries such as a secondary limb amputation. ${ }^{5}$ It is also important that previous incisions or tracts from biopsies or drains are completely excised in the definitive tumour resection. ${ }^{38}$

Adequate margins and oncological outcomes have been reported in STS of the extremities with vascular involvement treated with limbsparing surgery, with local recurrence rates ranging from 9-15\%.,8,31-34 STS with vascular involvement usually requires resection of the corresponding vessels. ${ }^{7}$ An exception is when only the aponeurosis of the vessel is invaded by the tumour, allowing tumour resection by sub-adventitial separation, which preserves the continuity of the great vessels. ${ }^{40}$ Due to the possibility of vessel damage caused by separation of the aponeuroses and the high risk of tumour contamination, sub-adventitial separation is regarded as an acceptable technique in selected cases such as lowgrade tumours that have invaded $<50 \%$ of the involved great vessel's diameter. ${ }^{41}$

When reconstructing a resected vessel, there is a choice between using autogenic vessels such as the great saphenous and femoral veins, allogenic vessels, or artificial vessels. ${ }^{7}$ The advantage of artificial vessels such as polytetrafluoroethylene and Dacron include a reduced duration of surgery and the avoidance of sampling morbidity as with autogenic vessel sampling. ${ }^{7}$ Artificial vessels can also be used when an autogenic vessel cannot be sampled and are more suitable than autogenic vessels when the vessel to be reconstructed has a large diameter. ${ }^{7}$ Artificial vessels are, however, associated with a high rate of infection. ${ }^{42}$ Allogenic vessels are also associated with a reduced duration of surgery compared to autogenic vessels, as well as a low incidence of complications. ${ }^{42}$ The long-term patency of reconstructed arteries is higher than that for venous reconstruction, with reports of patency rates between 60-100\%.,12,33,43 Venous reconstruction, on the other hand, remains controversial and depends on the bilateral status of venous return pre-operatively and on the residual venous return post-operatively. ${ }^{7}$ Both the tendency for some veins to become occluded early post-reconstruction and the high risk of chronic venous disease following limb-salvage surgery with extensive venous resection must be taken into consideration. ${ }^{7}$

Unlike vascular reconstruction, reconstruction of nerves involved in STS of the lower extremity does not guarantee preservation of function and remains controversial. ${ }^{7}$ Some authors do not advocate for the reconstruction of the sciatic nerve due to the prolonged duration of surgery and thus increased risk of post-operative complications such as delayed wound healing and infections, with no guarantee of preserving function. ${ }^{7,36}$ Autogenic nerves are the nerve grafts of choice when reconstructing nerves. ${ }^{7}$ Positive functional outcomes were shown in five patients undergoing autogenic common peroneal nerve reconstruction due to STS of the thigh with sciatic nerve involvement. ${ }^{44}$ All five patients recovered metatarsal sensation and could walk with the aid of an ankle brace. Further research regarding the 
regeneration potential of reconstructed sciatic nerves under the influence of chemotherapy and radiation therapy is necessary. ${ }^{36}$

The most commonly reported complications following limb-sparing surgery are infections, delayed wound healing, or wound dehiscence. ${ }^{20,45,46}$ The risk for these complications is higher with prolonged duration of surgery, patients over 40 years of age, and in the presence of neoadjuvant radiotherapy or chemotherapy. ${ }^{20,45,46} \mathrm{~A}$ wound morbidity rate of $34.4 \%$ has been reported by Skibber et al. ${ }^{45}$ in en bloc resections without adjuvant therapy. A similar wound morbidity rate of $37.0 \%$ was reported in a recent study in a cohort of 27 patients with lower limb STS and neural involvement who were treated with limbsparing surgery. ${ }^{15}$ Other complications of limbsparing surgery of the lower extremity include unplanned neurovascular injury, particularly in confined anatomical spaces such as the popliteal fossa, the formation of hematomas or seromas, devascularisation of soft tissue flaps, joint dislocations, and fractures. 15,20

The complexity of reconstructive limbsalvage surgery and its potential risks should be taken into consideration when planning surgical tumour resection. In cases where the risks of limb-salvage surgery outweigh the potential benefits in preserving limb function, an amputation may be more appropriate. This is especially relevant in distal lower limb STS, particularly of the foot, where amputation and early prosthetic fitting still have a role in the management of these tumours. ${ }^{47}$

\section{Chemotherapy}

The use of adjuvant chemotherapy is not considered a standard treatment in STS, but rather an individual and interdisciplinary decision, even in patients with an increased risk of developing metastatic disease. ${ }^{45}$ Local or distant disease recurrence and certain histopathologic entities such as synovial sarcomas or paediatric rhabdomyosarcomas are factors that favour the administration of adjuvant chemotherapy. ${ }^{5}$ Relative indications for adjuvant chemotherapy include high-grade, deeply located tumours larger than $5 \mathrm{~cm}$ in size, or intermediate-grade, deeply located tumours larger than $10 \mathrm{~cm}$ in size, especially in younger patients. ${ }^{48}$
Results from two meta-analyses of multiple randomised controlled trials point to an overall survival benefit of 5-10\% for adjuvant treatment with doxorubicin and ifosfamide. ${ }^{49,50}$ However, results of these studies need to be cautiously interpreted as pooled trials had conflicting results, posing an important limitation. Current guidelines from the National Comprehensive Cancer Network ${ }^{\circledR}$ (NCCN) as well as the European Society of Medical Oncology (ESMO) reflect on conflicting data as they refer to adjuvant chemotherapy as a legitimate option for high-risk STS but acknowledge the lack of consensus. ${ }^{21,51}$

In certain cases of STS with major neurovascular involvement, pre-operative chemotherapy, with the aim of local cytoreduction, may be a reasonable option, converting a potentially mutilating surgery to a less-invasive limbsparing operation. $.2,53$

\section{Radiation Therapy}

The goal of limb-sparing surgery is to achieve complete tumour resection with wide margins. Surgery as a single treatment modality for high-grade STS has, however, been associated with high local failure rates of $70-90 \% .^{54}$ Surgical resection has been demonstrated to be a sufficient single treatment modality in lowgrade subcutaneous STS of the extremities..$^{55}$ Baldini et $a .^{56}$ reported a local recurrence rate of $7 \%$ in a cohort of 74 patients with STS of the trunk or extremities with low- or intermediategrade small tumours managed with surgical resection alone, which is comparable with the reported local recurrence rates in STS treated with a combination of surgery and adjuvant radiotherapy.

The beneficial effects of adjuvant radiation therapy in patients with STS are generally well documented in the literature, with reported local control rates of $90 \%$ or greater. ${ }^{5}$ The development of neoadjuvant and adjuvant radiation therapy has led to comparable local control rates, even with focally positive marginal resections. ${ }^{57}$ Some studies have compared neoadjuvant radiation with post-operative radiation therapy and found no significant difference in local and distant disease control or disease-free survival. ${ }^{58,59}$ Several authors favour neoadjuvant radiation therapy as it has been associated with superior 
long-term functional outcomes compared to adjuvant therapy, especially in the lower extremity, despite the higher rates of wound complications. .,17,58 Flugstad et al. ${ }^{38}$ reported major wound complications in $18 \%$ of patients treated with adjuvant radiation therapy compared to an incidence of up to $37 \%$ in patients treated with neoadjuvant radiation therapy as reported by Bujko et al. ${ }^{60}$ Reported complications of adjuvant radiotherapy include an increased incidence of skin fibrosis, oedema, joint stiffness, and fractures. ${ }^{58}$ However, it has been suggested by some authors that radiation therapy is most effective when delivered to a low tumour load and should, therefore, be administered as adjuvant therapy, particularly in bulky tumours.' The dose of radiation has not been found to significantly influence local disease control. ${ }^{61}$ The use of brachytherapy in STS, which is usually administered through the insertion of a catheter over a 3-day period, has been shown to decrease local recurrence rates in some studies. ${ }^{62,63}$

Palliative radiotherapy for symptomatic relief is an important consideration in patients with advanced local and/or systemic disease. ${ }^{64}$

\section{FUTURE CONSIDERATIONS}

Schwarzbach et al. ${ }^{8}$ have proposed a classification system and treatment algorithm for STS of the extremities with vascular involvement, and a recent study has also classified STS of the lower extremity with sciatic nerve involvement. ${ }^{8,15}$ It is essential to validate the proposed classifications in prospective studies and assess the potential role of prognostic parameters such as tumour grade in further optimising the suggested treatment algorithms. Tumour grade is a recognised important prognostic factor in STS, which may influence the extent of surgical resection and any planned adjuvant therapies.1,15,61,65 The role of the histopathologic subtype of STS as an independent prognostic factor has also been previously emphasised by Pisters et al. ${ }^{65}$ Low-grade liposarcomas, for example, rarely metastasise and could potentially be treated with a nerve-sparing surgical resection, despite sciatic nerve encasement (Type A neural involvement), followed by adjuvant radiotherapy to reduce the risk of a local recurrence. ${ }^{15}$ The proposed vascular and neural classifications could also be combined into a single classification system for lower limb STS with neurovascular involvement..$^{15}$ For example, a tumour of the lower extremity with involvement of the femoral vein, no arterial involvement, and encasement of the sciatic nerve $<180^{\circ}$ would be classified as a Type IIIB STS and would be treated with an epineural dissection of the sciatic nerve, resection of the femoral vein, and potentially venous reconstruction if the collateral venous drainage is impaired.

\section{CONCLUSION}

Limb-sparing surgery has been established as the standard surgical treatment for lower limb STS, even in tumours with major neurovascular involvement. A multimodal treatment approach in a specialist centre is essential in treating these rare tumours, in addition to thorough preoperative assessment and planning. The use of classification systems in lower limb STS with neurovascular involvement can be integrated into the pre-operative planning of tumour resection, enabling a more precise treatment strategy.

\section{References}

1. Elias EG et al. Experience in the management of 52 patients with soft tissue sarcoma. The results of a median follow-up of seven years. $J$ Cancer Ther. 2008;6:47-54

2. Stiller CA et al. Descriptive epidemiology of sarcomas in Europe: report from the RARECARE project. Eur J Cancer. 2013;49(3):684-95.

3. Siegel R et al. Cancer statistics, 2014. CA Cancer J Clin. 2014;64(1):9-29.

4. Brennan MF et al. Lessons learned from the study of 10,000 patients with soft tissue sarcoma. Ann Surg. 2014;260(3):416-22.

5. Nystrom LM et al. Multidisciplinary management of soft tissue sarcoma. ScientificWorldJournal. 2013;2013:852462.

6. Thomas JE et al. Neurogenic tumors of the sciatic nerve. A clinicopathologic study of 35 cases. Mayo Clin Proc. 1983;58(10):640-7.

7. Lun DX et al. Management of great vessels and nerves in limbsalvage surgery for bone and soft tissue tumors. Orthop Surg. 2013;5(4):233-8.

8. Schwarzbach $\mathrm{MH}$ et al. Results of limb-sparing surgery with vascular replacement for soft tissue sarcoma in the lower extremity. J Vasc Surg. 2005;42(1):88-97.

9. Karakousis CP et al. Major vessel resection during limb-preserving surgery for soft tissue sarcomas. World J Surg. 1996;20(3):345-9

10. Ceraldi CM et al. Vascular reconstruction in the resection of soft 
tissue sarcomas. Perspect Vasc Surg Endovasc Ther. 2000;12(2):67-83.

11. Ghert MA et al. The surgical and functional outcome of limb-salvage surgery with vascular reconstruction for soft tissue sarcoma of the extremity. Ann Surg Oncol. 2005;12(12):1102-10.

12. Sternheim A et al. Space sarcomas: extra compartmental soft tissue tumors of the lower extremities a systematic approach to sarcomas of the femoral triangle, sartorial canal, and popliteal space. J Surg Oncol. 2009;99(5):281-91.

13. Tsukushi S et al. Results of limbsalvage surgery with vascular reconstruction for soft tissue sarcoma in the lower extremity: comparison between only arterial and arterovenous reconstruction. J Surg Oncol. 2008;97(3):216-20.

14. Brooks AD et al. Resection of the sciatic, peroneal, or tibial nerves: assessment of functional status. Ann Surg Oncol. 2002;9(1):41-7.

15. Sweiti $\mathrm{H}$ et al. Limb-salvage surgery of soft tissue sarcoma with sciatic nerve involvement. Sarcoma. 2018;2018:6483579.

16. Moser RP, Parish WM, "Radiologic evaluation of soft tissue tumors," Weiss SW, Goldblum JR (eds.) Enzinger and Weiss's Soft Tissue Tumors (2001) $4^{\text {th }}$ edition, Philadelphia: Mosby.

17. Pike $\mathrm{J}$ et al. Soft tissue sarcomas of the extremities: how to stay out of trouble. BCMJ. 2008;50(6):310-7.

18. Schwarzbach $\mathrm{MH}$ et al. Prognostic significance of preoperative [18F] fluorodeoxyglucose (FDG) positron emission tomography (PET) imaging in patients with resectable soft tissue sarcomas. Ann Surg. 2005;241(2):286-94.

19. Schwarzbach $\mathrm{MH}$ et al. Clinical value of [18-F] fluorodeoxyglucose positron emission tomography imaging in soft tissue sarcomas. Ann Surg. 2000;231(3):380-6.

20. Choong, PF. Principles of limb sparing surgery in bone and soft tissue sarcoma. Cancer Forum. 2010;34(3):145-8.

21. ESMO/European Sarcoma Network Working Group. Soft tissue and visceral Sarcomas: ESMO Clinical Practice Guidelines for diagnosis, treatment and follow-up. Ann Oncol. 2014;25(Suppl 3):102-12.

22. Mankin HJ, Hornicek FJ. Diagnosis, classification, and management of soft tissue sarcomas. Cancer Control. 2005;12(1):5-21.

23. Mankin $\mathrm{HJ}$ et al. The hazards of the biopsy, revisited. Members of the Musculoskeletal Tumor Society. J Bone Joint Surg Am. $1996 ; 78(5): 656-63$
24. Simon MA, Biermann JS. Biopsy of bone and soft-tissue lesions. J Bone Joint Surg Am. 1993;75(4):616-21.

25. Conrad EU et al. "Pelvis," Simon MA, Springfield D (eds.) Surgery for Bone and Soft Tissue Tumors (1998) Philadelphia: Lippincott-Raven, pp.323-41.

26. O'Sullivan B, Pisters PW. Staging and prognostic factor evaluation in soft tissue sarcoma. Surg Oncol Clin N Am. 2003;12(2):333-53.

27. Noria $\mathrm{S}$ et al. Residual disease following unplanned excision of soft-tissue sarcoma of an extremity. J Bone Joint Surg Am. 1996;78(5):650.

28. Giuliano AE et al. The management of locally recurrent soft-tissue sarcoma. Ann Surg. 1982;196(1):87-91.

29. Rosenberg SA et al. The treatment of soft-tissue sarcomas of the extremities: prospective randomized evaluations of (1) limb-sparing surgery plus radiation therapy compared with amputation and (2) the role of adjuvant chemotherapy. Ann Surg. 1982;196(3):305-15.

30. Nambisan RN et al. Malignant soft tissue tumors of nerve sheath origin. J Surg Oncol. 1984;25(4):268-72.

31. Koperna T et al. Vascular reconstruction for limb salvage in sarcoma of the lower extremity. Arch Surg. 1996;131(10):1103-7.

32. Nambisan RN, Karakousis CP. Vascular reconstruction for limb salvage in soft tissue sarcomas. Surgery. 1987;101(6):668-77.

33. Leggon RE et al. Limb salvage surgery with vascular reconstruction. Clin Orthop Relat Res. 2001;387:207-16.

34. Williard WC et al. Comparison of amputation with limb-sparing operations for adult soft tissue sarcoma of the extremity. Ann Surg. 1992;215(3):269-75.

35. Bickels $J$ et al. Sciatic nerve resection: is that truly an indication for amputation? Clin Orthop Relat Res. 2002;399:201-4.

36. Fuchs B et al. Sciatic nerve resection in the thigh: a functional evaluation. Clin Orthop Relat Res. 2001;382:34-41.

37. Clarkson PW et al. Epineural dissection is a safe technique that facilitates limb salvage surgery. Clin Orthop Relat Res. 2005;438:92-6.

38. Flugstad DL et al. Importance of surgical resection in the successful management of soft tissue sarcoma. Arch Surg. 1999;134(8):856-61.

39. Gerrand $\mathrm{CH}$. Classification of positive margins after resection of soft-tissue sarcoma of the limb predicts the risk of local recurrence. J Bone Joint Surg Br. 2001;83(8):1149-55.

40. Wang $Z$ et al. Functional outcomes and complications of reconstruction of the proximal humerus after intra- articular tumor resection. Orthop Surg. 2010;2(1):19-26.

41. Fortner JG et al. Limb-preserving vascular surgery for malignant tumors of the lower extremity. Arch Surg. 1977;112(4):391-4.

42. Faenza A et al. Vascular homografts for vessel substitution in skeletal and soft tissue sarcomas of the limbs. Transplant Proc. 2005;37(6):2692-3.

43. Nishinari $\mathrm{K}$ et al. Vascular reconstruction in limbs associated with resection of tumors. Ann Vasc Surg. 2003;17(4):411-6.

44. Melendez $M$ et al. Sciatic nerve reconstruction: limb preservation after sarcoma resection. Ann Plast Surg. 2001;46(4):375-81.

45. Skibber JM et al. Limb-sparing surgery for soft tissue sarcomas: wound related morbidity in patients undergoing wide local excision. Surgery. 1987;102(3):447-52.

46. Arbeit JM et al. Wound complications in the multimodality treatment of extremity and superficial truncal sarcomas. J Clin Oncol. 1987;5(3):480-8.

47. Ferguson PC. Surgical considerations for management of distal extremity soft tissue sarcomas. Curr Opin Oncol. 2005:17(4):366-9.

48. Sarcoma Meta-analysis Collaboration. Adjuvant chemotherapy for localised resectable soft-tissue sarcoma of adults: meta-analysis of individual data. Lancet. 1997;350(9092):1647-54.

49. American Academy of Orthopaedic Surgeons. "Musculoskeletal tumours 2," Orthopaedic Knowledge Update (2007) Illinois: Rosemont.

50. Pervaiz $\mathrm{N}$ et al. A systematic metaanalysis of randomized controlled trials of adjuvant chemotherapy for localized resectable soft-tissue sarcoma. Cancer. 2008;113(3):573-81.

51. von Mehren M et al. Soft tissue sarcoma, version 2.2014. J Nat Compr Canc Netw. 2014;12(4):473-83.

52. Casali PG. Adjuvant chemotherapy for soft tissue sarcoma. Am Soc Clin Oncol Educ Book. 2015:629-33.

53. Baldini EH et al. Neoadjuvant chemotherapy, concurrent chemoradiation, and adjuvant chemotherapy for high-risk extremity soft tissue sarcoma. Am Soc Clin Oncol Educ Book. 2018;38:910-5.

54. Lindberg RD. Treatment of localized soft tissue sarcomas in adults at M.D. Anderson Hospital and Tumor Institute. Cancer Treat Symp. 1985;3:59-65

55. Gibbs CP et al. Oncological outcomes of operative treatment of subcutaneous soft-tissue sarcomas of the extremities. J Bone Joint Surg Am. 1997;79(6):888-97. 
56. Baldini EH et al. Long-term outcomes after function-sparing surgery without radiotherapy for soft tissue sarcoma of the extremities and trunk. J Clin Oncol. 1999;17(10):3252-9.

57. Dagan $\mathrm{R}$ et al. The significance of a marginal excision after preoperative radiation therapy for soft tissue sarcoma of the extremity. Cancer. 2012;118(12):3199-207.

58. O'Sullivan B et al. Preoperative versus postoperative radiotherapy in soft-tissue sarcoma of the limbs: a randomised trial. Lancet. 2002;359(9325):2235-41.
59. Cheng EY et al. Soft tissue sarcomas: preoperative versus postoperative radiotherapy. J Surg Oncol.

1996;61(2):90-9.

60. Bujko $\mathrm{K}$ et al. Wound healing after preoperative radiation for sarcoma of soft tissues. Surg Gynecol Obstet. 1993:176(2):124-34.

61. Herbert $\mathrm{SH}$ et al. Limb-preserving treatment for soft tissue sarcomas of the extremities. The significance of surgical margins. Cancer. 1993;72(4):1230-8.

62. Clarke DH, Martinez A. An overview of brachytherapy in cancer management. Oncology (Williston Park). 1990;4(9):39-46.
63. Schray MF et al. Soft tissue sarcoma integration of brachytherapy, resection, and external irradiation. Cancer. 1990;66(3)451-56.

64. Tween $\mathrm{H}$ et al. Radiotherapy for the palliation of advanced sarcomasthe effectiveness of radiotherapy in providing symptomatic improvement for advanced sarcomas in a single centre cohort. Healthcare (Basel). 2019;7(4):120.

65. Pisters PW et al. Analysis of prognostic factors in 1,041 patients with localized soft tissue sarcomas of the extremities. J Clin Oncol. 1996:14(5):1679-89. 